\title{
APPLICATIONS
}

\section{Tracking and back-tracking}

\author{
E. Lorenzo* , L. Narvarte and J. Muñoz \\ Instituto de Energía Solar, Grupo de Sistemas Fotovoltaicos, Universidad Politécnica de Madrid (IES-UPM), Ciudad Universitaria s/n, \\ 28040 Madrid, Spain
}

\begin{abstract}
This paper presents a review of back-tracking geometry not only for single axis but also for two-axis tracking and analyses the corresponding energy gains. It compares the different back-tracking strategies with the ideal tracking in terms of energy yield concluding, on the one hand, that back-tracking is more useful for single horizontal axis than for the single vertical one, and on the other hand, that back-tracking is more efficient when applied in the primary axis of a two-axis tracker.
\end{abstract}

\section{KEYWORDS}

tracking; back-tracking

\section{*Correspondence}

E. Lorenzo, Instituto de Energía Solar, ETSI Telecomunicación, Ciudad Universitaria s/n, 28040 Madrid, Spain. E-mail: lorenzo@ies-def.upm.es

\section{INTRODUCTION}

Solar tracking is been used with PV flat modules for more than 20 years, and some studies [1] suggest that it will maintain its interest while the PV module cost remains more than $0.8 € / \mathrm{Wp}$. More than 20 companies currently offer products with different tracking possibilities: two axis, single vertical axis, single horizontal axis and single tilted axis [2-5]. When several trackers are placed in a limited area, the problem of mutual shadowing arises. A previous paper [1] has dealt with trackers on the assumption of ideal tracking, i.e. when all the receiver surfaces are always perpendicular to the Sun. Tracking geometry has been reviewed and the relationship between the yearly energy gain and land occupation has been analysed.

Shade not only reduces the electric output power but also increases the risk of hot spots. Hence, the interest of the so called back-tracking strategy, which consists of moving the surface angles away from then ideal values, just enough to get the shadow borderline to pass outside the border of the adjacent tracker. This way, first, shade is fully avoided and, second, the loss due to the angle of incidence is minimised. Back-tracking geometry for single horizontal [6] and single vertical axis [7] was described some years ago. This paper presents a comprehensive review of back-tracking geometry not only for single axis but also for two-axis tracking, and analyses the associated energy gains. The results help us to understand why backtracking implementation in commercial products is today restricted to single horizontal axis trackers, and suggest that back-tracking is still attractive for other trackers, in particular for the two axis ones.

\section{SINGLE HORIZONTAL AXIS}

Figure 1a describes the geometry of a single horizontal, $\mathrm{N}-\mathrm{S}$ oriented axis, ideal tracking in the case of mutual shadowing. The ideal tracking angle, $\omega_{\mathrm{ID}}$, is given by:

$$
\tan \omega_{\mathrm{ID}}=\frac{x}{z}
$$

where $(x, y, z)$ are the Cartesian coordinates of the Sun vector referred to a system with the $x$-axis pointing west, the $y$-axis pointing south and the $z$-axis pointing to the zenith [1]. These coordinates are related to solar elevation, $\gamma_{\mathrm{s}}$, and solar azimuth, $\psi_{\mathrm{s}}$, angles by:

$$
\begin{aligned}
& x=\cos \gamma_{\mathrm{S}} \sin \psi_{\mathrm{S}} \\
& y=\cos \gamma_{\mathrm{S}} \cos \psi_{\mathrm{S}} \\
& z=\sin \gamma_{\mathrm{S}}
\end{aligned}
$$

Shadow length, $s$, and shaded fraction of the PV generator area, FS, are given by:

$$
\begin{gathered}
s=\frac{1}{\cos \omega_{\mathrm{W}}} \\
\mathrm{FS}=\max \left[0,\left(1-\frac{L_{\mathrm{EW}}}{s}\right)\right]
\end{gathered}
$$


(a)

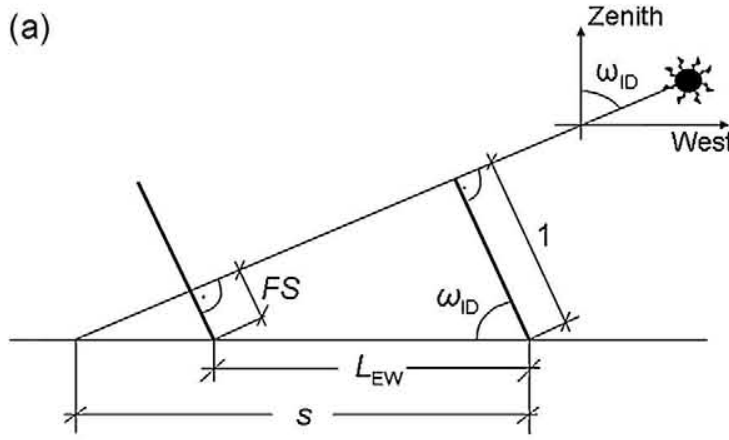

(b)

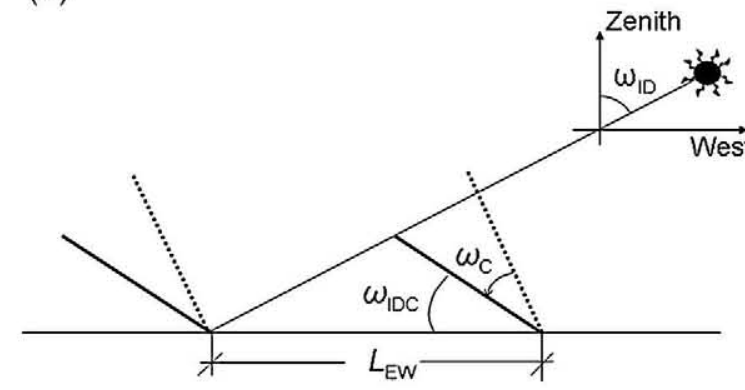

Figure 1. Geometry of a single horizontal, N-S oriented, axis tracking. Projections on the vertical, E-W oriented, plane: (a) ideal tracking in the case of shadowing, (b) back-tracking.

where $L_{\mathrm{EW}}$ is the distance between axes. The back-tracking correction angle, $\omega_{\mathrm{C}}$, is:

$$
\begin{gathered}
\omega_{\mathrm{C}}=\omega_{\mathrm{ID}}-\omega_{\mathrm{IDC}} \\
\cos \omega_{\mathrm{C}}=L_{\mathrm{EW}} \cos \omega_{\mathrm{ID}}
\end{gathered}
$$

It is worth mentioning that the ratio between the PV array area to total ground area, the so-called ground cover ratio (GCR), is given by:

$$
\mathrm{GCR}=\frac{1}{L_{\mathrm{EW}}}
$$

Now, let us consider a PV array affected by shading. At any instant, we can state:

$$
P_{\mathrm{S}}=P_{\mathrm{NS}}\left(1-F_{\mathrm{ES}}\right)
$$

where $P_{\mathrm{S}}$ and $P_{\mathrm{NS}}$ represent the power delivered by the PV array with and without shading, respectively, and $F_{\mathrm{ES}}$ known here as the effective shading factor, whose value determines the power decrease and depends on the particular electric PV system layout (mainly solar cells series and parallel association, and bypass diode disposition). Whatever the case, it encompasses two extremes. The most optimistic is when the power reduction is just equal to the shaded fraction, FS, that is:

$$
F_{\mathrm{ES}}=\mathrm{FS}
$$

And the most pessimistic is when the mere existence of any shade fully cancels out the power. That is:

$$
\mathrm{FS}>0 \Rightarrow F_{\mathrm{ES}}=1
$$

A better approximation is obtained by taking into account the shaded blocks. A 'block' is defined here as a group of cells protected by one bypass diode. A block is shaded when at least one of its cells is shaded. An accurate empirical expression is [8]:

$$
\left(1-F_{\mathrm{ES}}\right)=(1-\mathrm{FS})\left(1-\frac{N_{\mathrm{SB}}}{N_{\mathrm{TB}}+1}\right)
$$

where $N_{\mathrm{TB}}$ is the total number of blocks inside the specific array and $N_{\mathrm{SB}}$ is the number of shaded blocks. In that follows, the cases defined by Equations (8)-(10) will be termed as 'optimistic', 'pessimistic' and 'realistic', respectively.

In order to analyse the back-tracking energetic impact, we have calculated the energy yield of grid connected PV systems, with and without back-tracking, for the two different representative electric layouts described in Figure 2a,b. The PV module (Figure 2c) is made up of 48 solar cells and 3 bypass-diodes, arranged in such a way that the number of blocks is $3 \times 1$ (horizontal $\times$ vertical). Then, layout Figure 2a,b are characterised, respectively, by $90 \times 1$ and $90 \times 2$ blocks. Calculations have been made with the IES-UPM own code, whose details can be found in Reference [1]. This code considers the effect of changing absorptance of the PV module with incidence angle and also the effect of albedo. The selected location is Amareleja, in South Portugal (Latitude: 38.2N; Longitude: $7.2 \mathrm{~W}$; solar radiation and ambient temperature data as given by PVGIS [9]). Despite not being directly related with this paper, it is worth mentioning that there has been a $48 \mathrm{MW}$ PV plant in this village, based on trackers, in routine operation since 2009 (http://www.acciona-energia.com). Figure 3 shows the evolution of the yearly energy yield versus distance between tracker axes, or 1/GCR. Table I gives numerical results for $1 / \mathrm{GCR}=3.5$, often found in practical applications. The key result is that, compared with ideal tracking, the back-tracking strategy is always close to the optimistic case and better than the realistic ones. Therefore, back-tracking is highly attractive, which helps us to understand why it has been implemented in single horizontal axis trackers for many years [10,11]. It is worth mentioning that PV modules at Figure 2 are oriented in such a way that their short side is parallel to the rotation axis. In fact, because the bypass disposition of these particular PV modules, the orientation with their long side parallel to the axis encompasses less shading sensitivity. However, back-tracking is still attractive also in this case.

\section{SINGLE TILTED AND DEVIATED AXIS}

The geometry of back-tracking for a rotating single axis, tilted an angle $\beta_{\text {AxIs }}$ (the angle that the axis makes with the horizontal plane) and aligned at an angle $\alpha_{\mathrm{AxIS}}$ relative to the N-S direction is an analogue to the previous case. It must be noted that the coordinates of the Sun $\left(x^{\prime}, y^{\prime}, z^{\prime}\right)$, 


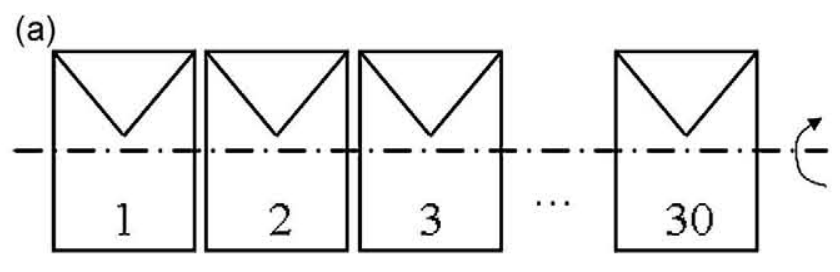

(b)

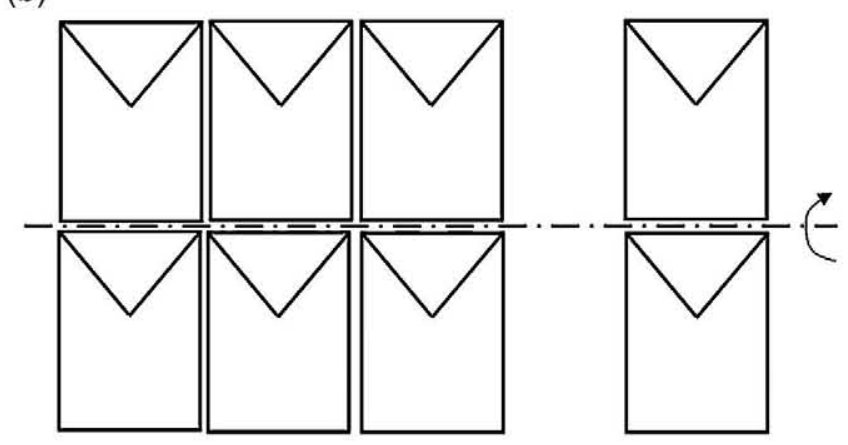

(c)

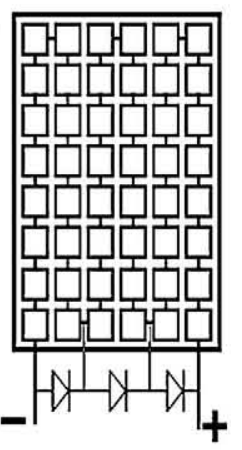

Figure 2. Electrical layouts of two different PV generators, (a) and (b), whose module has 48 solar cells and three bypass-diodes (c).

now referred to as a system with the $y$-axis coinciding with the rotating tracking axis; the $x$-axis horizontal, roughly pointing west and the $z$-axis pointing upwards, are given by:

$$
\begin{gathered}
x^{\prime}=x \cos \alpha_{\mathrm{AXIS}}-y \sin \alpha_{\mathrm{AXIS}} \\
y^{\prime}=x \cos \beta_{\mathrm{AXIS}}+y \cos \beta_{\mathrm{AXIS}} \cos \alpha_{\mathrm{AXIS}}-z \sin \beta_{\mathrm{AXIS}} \\
z^{\prime}=x \sin \beta_{\mathrm{AXIS}} \sin \alpha_{\mathrm{AXIS}}+y \sin \beta_{\mathrm{AXIS}} \cos \alpha_{\mathrm{AXIS}}+z \cos \beta_{\mathrm{AXIS}}
\end{gathered}
$$

Figure 4 represents the energy yield for the same cases as Figure 2, but now with the axes tilted $\beta_{\mathrm{AXIS}}=20^{\circ}$ and deviated also at $\alpha_{\text {AXIS }}=20^{\circ}$. Table I gives numerical results for $1 / \mathrm{GCR}=3.5$. Again, back-tracking is close to the optimistic case and better than the realistic cases. It is worth mention that deviated axes, despite being not optimal, offer sometimes practical interest for adapting to non-flat terrains.

\section{SINGLE VERTICAL AXIS}

Let us consider a set of vertical trackers arranged as shown in Figure 5. A general tracker (like the striped one, in the centre of the figure) can be shaded from five other trackers, respectively placed at its E, SE, S, W and WE. Of course, it is possible to also imagine shades from other than these five trackers. For example, from ones placed at ESE and WSW. In higher latitudes shading can likely also occur from NE and NW trackers. However, these cases encompassed rather limited practical interest, so we have decided to avoid here the associated complexity.
Figure 6 shows a case of shading from West. Ideal tracking angle, shadow length and shaded fraction are given by:

$$
\begin{gathered}
\tan \omega_{\mathrm{ID}}=\frac{x}{y} \\
s=b\left(\cos \beta_{\mathrm{AXIS}}+\sin \beta_{\mathrm{AXIS}} \cot \gamma_{\mathrm{S}}\right) \\
\mathrm{FS} S_{\mathrm{W}}=\mathrm{FS} 1_{\mathrm{W}} \mathrm{FS} 2_{\mathrm{W}}
\end{gathered}
$$

Where:

$$
\begin{aligned}
\mathrm{FS} 1_{\mathrm{W}} & =\frac{s-L_{\mathrm{EW}} \sin \psi_{\mathrm{S}}}{s} \text { and } \mathrm{FS} 2_{\mathrm{W}} \\
& =\frac{1-L_{\mathrm{EW}} \cos \psi_{\mathrm{S}}}{1}
\end{aligned}
$$

The back-tracking correction angle is again given by Equations (4) and (5) ${ }^{1}$. Obviously, shading from $\mathrm{E}$ is symmetric to this case.

Figure 7 show a case of shading from SW. Shaded fraction is now given by:

$$
\mathrm{FS}_{\mathrm{SW}}=\mathrm{FS} 1_{\mathrm{SW}} \mathrm{FS} 2_{S W}
$$

Where:

$$
\begin{aligned}
& \mathrm{FS} 1_{\mathrm{SW}}=1-\frac{L_{\mathrm{NS}} \cos \psi_{\mathrm{S}}+L_{\mathrm{EW}} \sin \psi_{\mathrm{S}}}{s} \quad \text { and } \\
& \mathrm{FS} 2_{\mathrm{SW}}=\frac{\tan \psi_{\mathrm{S}}-L_{\mathrm{EW}} / L_{\mathrm{NS}}}{\left(L_{\mathrm{EW}}+\cos \psi_{\mathrm{S}}\right)\left(L_{\mathrm{NS}}-\sin \psi / t_{\mathrm{S}}\right)-L_{\mathrm{EW}} / L_{\mathrm{NS}}}
\end{aligned}
$$

It is also possible to establish a back-tracking strategy for avoiding shade from the SE and SW trackers. However,

${ }^{1}$ In fact, Equation (5) is the solution for the particular case $\beta_{\mathrm{AXIS}}=\pi / 2$, i.e. with the tracker vertical. But, it can be accepted as a general solution without great error. The interested reader is encouraged to consult the reference [7]. 


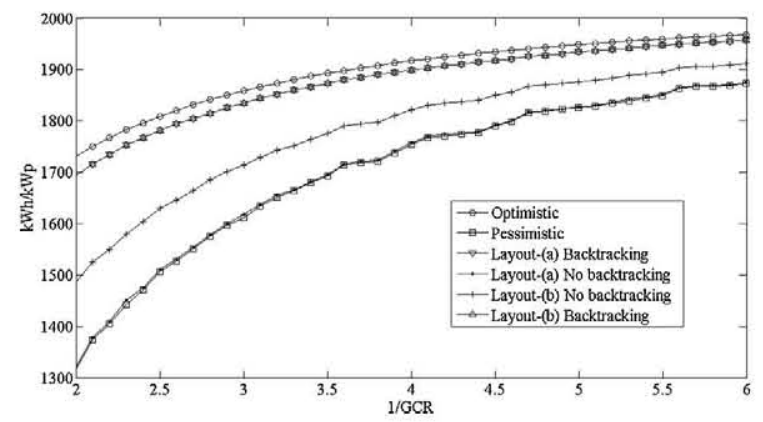

Figure 3. Yearly energy yield versus the distance between axes for single horizontal axis tracking. Back-tracking is close to the optimistic cases and better than the two realistic cases.

this would be scarcely practical, because it encompasses a sudden $180^{\circ}$ turn. For example, back-tracking for avoiding SW shade forces trackers to move towards the SE until they, shade and shaded, are precisely aligned with the Sun. Just at this moment, the trackers should turn $180^{\circ}$. Otherwise, The Sun's rays will fall on the back surface of the PV modules. Apart of that, because shading occurrence is normally associated to low Sun elevation angles, shading from the SE and SW used to be scarcely relevant. For example, for the case considered in Table I (described below) energy losses due to shading from the $\mathrm{E}$ and $\mathrm{W}$, and $\mathrm{SE}$ and SW are 8 and $0.4 \%$, respectively. For both reasons, lack of practical sense and irrelevance have led us to disregard the implementation of back-tracking to avoid shading from SE and SW. Along the same lines, we have also disregard designs involving any shading from $\mathrm{S}$. It is easy to see that this leads to the condition:

$$
\frac{L_{\mathrm{NS}}}{b} \geq \cos \beta_{\mathrm{AXIS}}+\sin \beta_{\mathrm{AXIS}} \tan \left(\phi+\delta_{\mathrm{MAX}}\right)
$$

Where $\phi$ is the site latitude and $\delta_{\mathrm{MAX}}=23.5^{\circ}$ the maximum Sun declination angle.
Figure 8 shows energy yield versus the GCR for a single vertical axis tracking, again at Amareleja. The geometry is defined by the $\beta_{\mathrm{AXIS}}=40^{\circ}, b=1 / \sqrt{2}, L_{\mathrm{NS}}=2.06$; and electric layout by the number of bypass diodes in the horizontal and vertical direction of each tracker, 18 and 3, respectively. Table I gives numerical results for 1/ $\mathrm{GCR}=4.5$. Now, the key result is that, compared with ideal tracking, back-tracking strategy is slightly better than the realistic case and both are close to the optimistic case. Obviously, this result is dependent on the particular geometry and electric layout. In fact, as the number of bypass diodes in the vertical direction increases, shading losses decrease and, therefore, back-tracking becomes less attractive. However the sensitivity of energy to layout is in practice rather low. For example, changing the electrical layout from $18 \times 3$ to $18 \times 6$ led to reduce shading losses by about $1 \%$. Hence, one can say that back-tracking on vertical axis tracking tends to be roughly neutral in pure energy terms. That helps us to understand why it is not implemented in today's commercial vertical axis trackers. However, it must be noted that shade is prone to inducing hot-spots, which can even result in PV module destruction after a certain operating time [12] and that back-tracking fully avoids shade. Hence, even irrespective of the energy balance, we think back-tracking can still be attractive in practise.

\section{TWO AXES, PRIMARY VERTICAL}

Two-axis trackers are made up of an axis connected to the foundation, called the primary axis by us, and another axis fixed to the primary, here called the secondary. The primary vertical axis, or pedestal, is today the most common twoaxis tracker. The figures and equations above for the single vertical axis can also be applied here, once it is considered that the $\beta_{\mathrm{AXIS}}$ is now continuously adjusted by rotating the

Table I. Yearly energy yield of grid-connected PV systems for several tracking types, with and without back-tracking, in a particular location.

\begin{tabular}{|c|c|c|c|c|c|}
\hline \multirow[t]{2}{*}{ Tracking type } & \multicolumn{3}{|c|}{ Single axis } & \multicolumn{2}{|c|}{ Two axes } \\
\hline & Horizontal N-S & Tilted, $20^{\circ}$ & Vertical & Primary vertical & Primary horizontal \\
\hline $1 / \mathrm{GCR}$ & 3.5 & 3.5 & 4.5 & 5.5 & \\
\hline \multicolumn{6}{|l|}{ Without back-tracking } \\
\hline Optimistic & 1893 & 2071 & 2132 & 2316 & 2225 \\
\hline Realistic (a) & $1776(6.2)$ & $1919(7.3)$ & $2121(0.5)$ & $2302(0.6)$ & $2124(4.5)$ \\
\hline Realistic (b) & 1695 (10.5) & $1878(9.3)$ & & & $2103(5.5)$ \\
\hline Pessimistic & $1695(10.5)$ & $1919(7.3)$ & $2086(2.2)$ & $2263(2.3)$ & \\
\hline \multicolumn{6}{|l|}{ With back-tracking } \\
\hline On the primary axis & $1873(1.0)$ & $2054(0.8)$ & $2129(0.14)$ & $2315(0.04)$ & $2217(0.3)$ \\
\hline On the secondary axis & & & & $2276(1.7)$ & $2124(4.5)$ \\
\hline On both axes & & & & $2287(1.2)$ & $2217(0.3)$ \\
\hline
\end{tabular}

Values are given in $\mathrm{kWh}$ per $\mathrm{kW}$ of PV generator peak power. The numbers given in parentheses as percentages represent, the losses with respect to the optimistic cases. It must be noted that, despite being set here to $100 \%$, these optimistic cases still include some shading losses due to mutual shading between trackers. For example, the energy yield with only one single axis tracker $(1 / \mathrm{GCR} \rightarrow \infty)$ is 2325 , so the optimistic case as defined here includes $8.3 \%$ shading losses. 


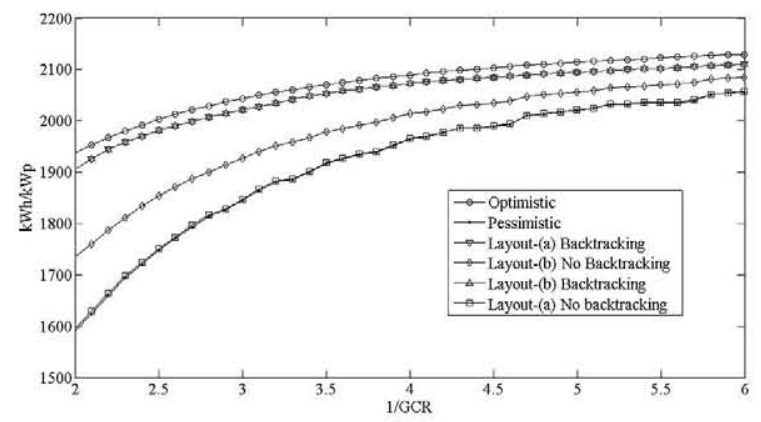

Figure 4. Yearly energy yield versus the distance between axes for single axis tracking tilted $\beta_{\mathrm{AXIS}}=20^{\circ}$ and deviated also at $\alpha_{\text {AXIS }}=20^{\circ}$. The back-tracking is, again, close to the optimistic cases and better than the two realistic cases

secondary axis in such a way that:

$$
\begin{gathered}
\beta_{\mathrm{AXIS}}=\beta_{\mathrm{ID}} \\
\tan \beta_{\mathrm{ID}}=\frac{\left(x^{2}+y^{2}\right)}{z}
\end{gathered}
$$

As regards back-tracking, two axes imply two different possibilities. For example, shading from the West, described in Figure $6 \mathrm{a}$, can be avoided by rotating the primary axis towards S (Figure 6b), Equations (4) and (5) or by rotating the secondary axis towards the horizontal, to a tilt angle, $\beta_{\mathrm{IDC}}$. In this case, the back-tracking correction
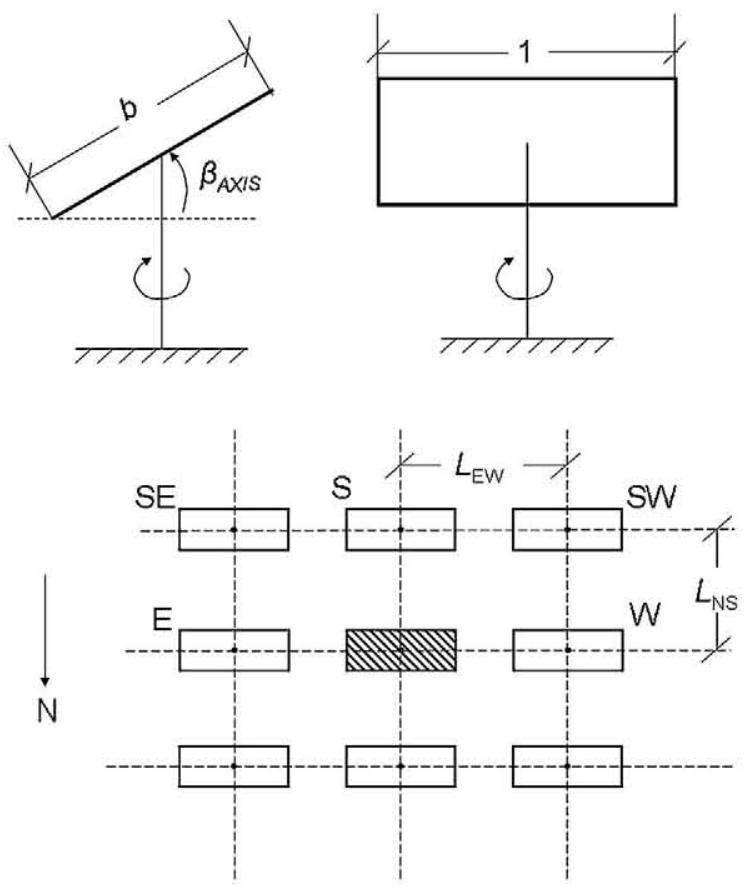

Figure 5. Geometry of a field with single vertical axis trackers: tilt angle, $\beta_{\text {AxIS; }}$ aspect relation $b$; spacing between adjacent trackers in the North-South direction and East-West direction, $L_{N S}$ and $L_{E W}$. A general tracker (in the centre of the figure) can receive shade from five trackers, placed at the $E, S E, S, S W$ and $W$.

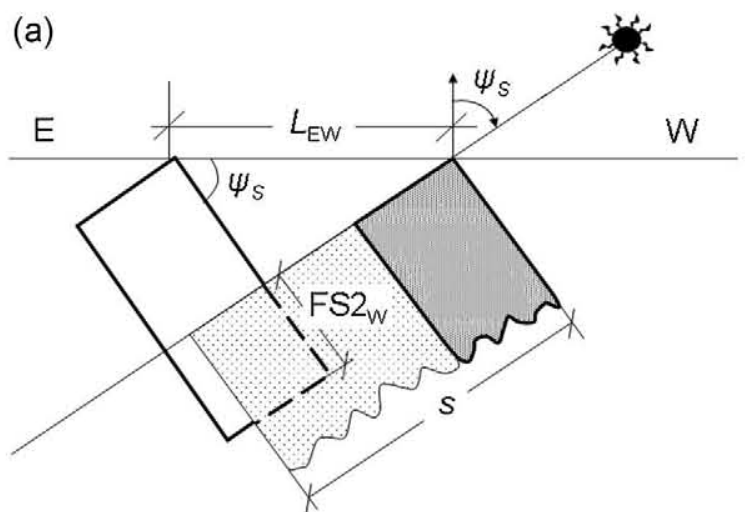

(b)

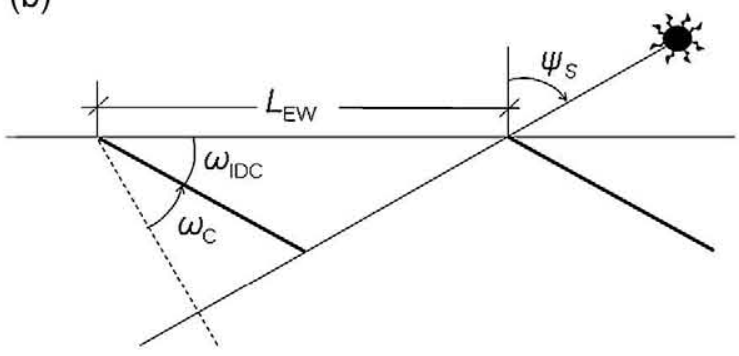

Figure 6. Geometry of single vertical axis tracking. Projections on the horizontal plane: (a) ideal tracking in a case of shading from West, (b) corresponding back-tracking

angle is:

$$
\begin{gathered}
\beta_{\mathrm{C}}=\beta_{\mathrm{ID}}-\beta_{\mathrm{IDC}} \\
\cos \beta_{\mathrm{IDC}}=\frac{L_{\mathrm{EW}}}{b} \cos \beta_{\mathrm{ID}}
\end{gathered}
$$

Shading from the $\mathrm{SW}$ can also be avoided by rotating the secondary axis towards the horizontal. Now, the back-

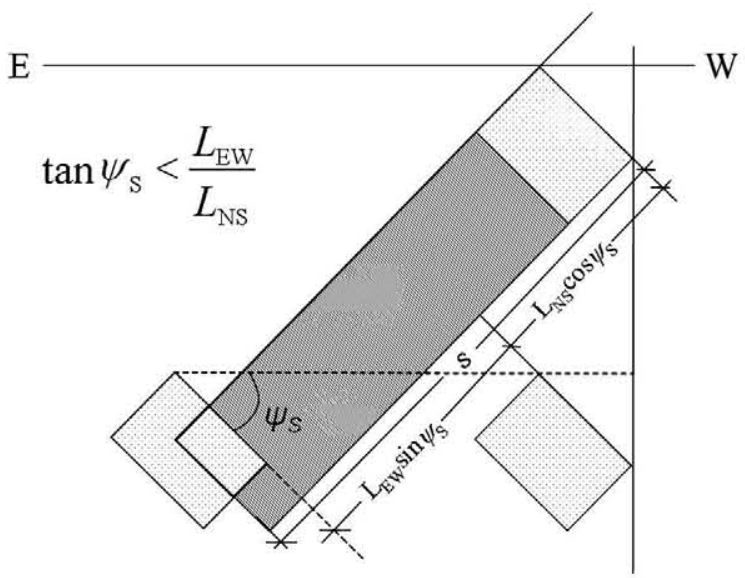

Figure 7. Geometry of single vertical axis tracking. Projections on the horizontal plane: ideal tracking in a case of shading from South-West. 


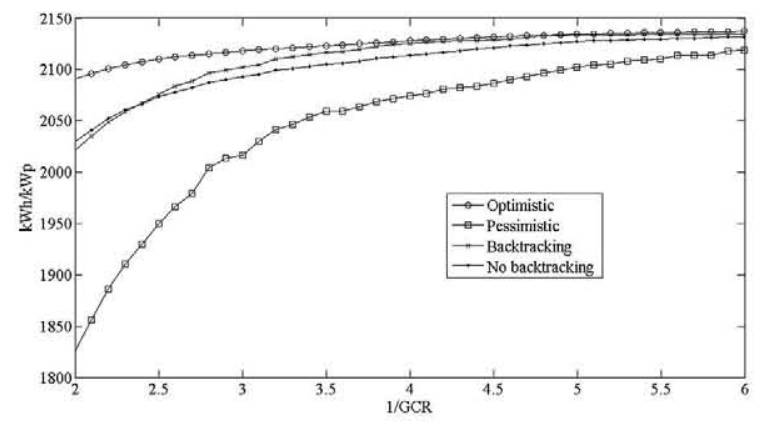

Figure 8. Energy yield versus the GCR for a single vertical axis tracking at Amareleja.

tracking correction angle is:

$$
\cos \beta_{\mathrm{IDC}}=\frac{L_{\mathrm{EW}} \sin \psi_{\mathrm{S}}+L_{\mathrm{NS}} \cos \psi_{\mathrm{S}}}{b} \cos \beta_{\mathrm{ID}} s
$$

Figure 9 shows energy yield versus the GCR for a twoaxis tracking, primary vertical, again at Amareleja and with $b=1 / \sqrt{ } 2, L_{\mathrm{NS}}=2.06$ and $N_{\mathrm{TB}}=18 \times 3$. The tilt angle has been limited to $75^{\circ}$. Table I gives numerical results for $1 /$ GCR $=5.5$. Three different back-tracking possibilities have been considered: only on the primary, or vertical, axis (this way, only shade from the $\mathrm{E}$ and $\mathrm{W}$ are avoided); only on the secondary, or horizontal, axis (all shade, from the E, W, SE and SW are avoided) and on the two axes, each time selecting the axis requiring the lowest back-tracking correction angle (all shade is again avoided). Now, the key result is that the back-tracking is significantly more efficient on the primary axis than on the secondary one.

\section{TWO AXES, PRIMARY HORIZONTAL}

Figure 10 shows the geometry of a two-axis tracking, the primary being horizontal, in a case of mutual shadowing. This kind of tracking has led to several commercial products (see, for e.g. [13]). The ideal primary axis rotating angle is given by Equation (1). The ideal secondary axis tilt angle, $\beta_{\mathrm{ID}}$, shaded

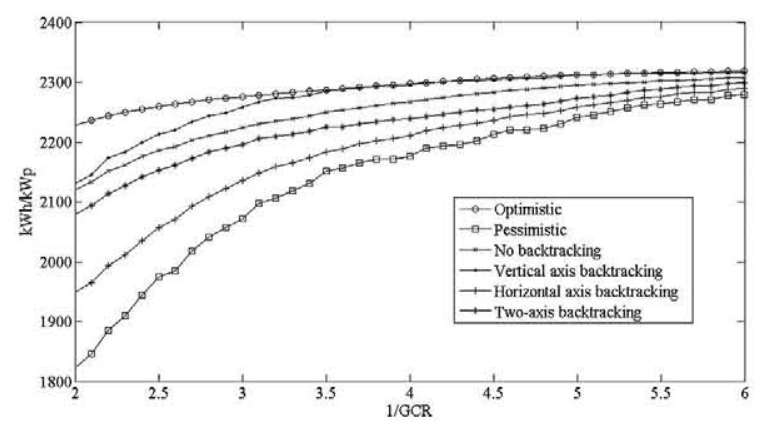

Figure 9. Energy yield versus the GCR for a two-axis tracking, primary vertical at Amareleja with $b=1 / \sqrt{ } 2, \mathrm{LNS}=2.06$ and $\mathrm{NTB}=18 \times 3$.
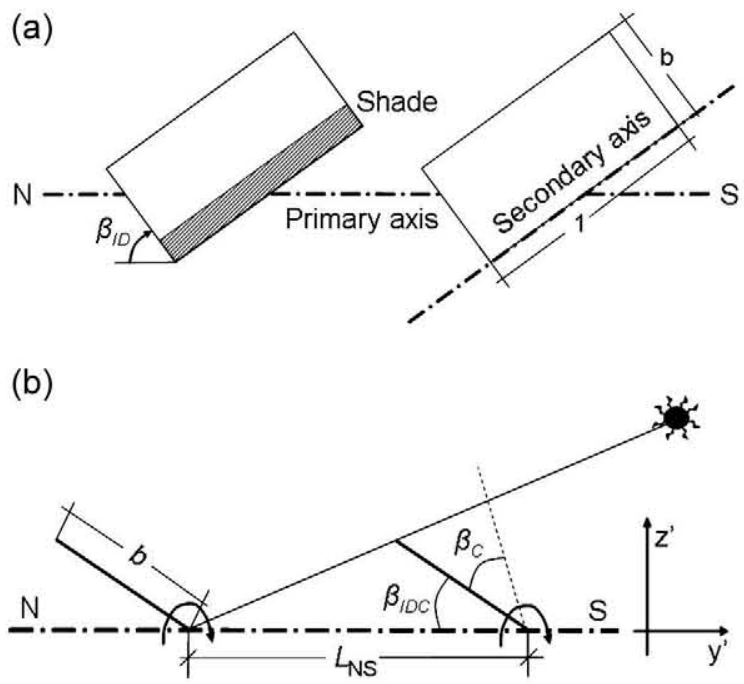

Figure 10. Geometry of a two-axis tracking, being the primary horizontal, in a case of mutual shadowing.

fraction and back-tracking correction angle are given by:

$$
\begin{aligned}
& \tan \beta_{\mathrm{ID}}=\frac{y}{\left(x^{2}+z^{2}\right)^{1 / 2}} \\
& \mathrm{FS}_{\mathrm{N}}=1-\frac{L_{\mathrm{NS}}}{\left(b / \cos \beta_{\mathrm{ID}}\right)} \\
& \cos \beta_{\mathrm{IDC}}=\frac{L_{\mathrm{NS}}}{b} \cos \beta_{\mathrm{ID}}
\end{aligned}
$$

It is worth commenting that, during the extreme hours of the day, back-tracking on the primary axis - again, Equations (4) and (5) - affects the value of the tilt angles (ideal and back-tracking) of the secondary axis. Corresponding equations can be derived from geometric considerations. In order to make the understanding of the paper easier, these equations are not presented here, but they have been implemented in the corresponding software code.

Figure 11 shows energy yield versus the GCR for a twoaxis tracking, primary horizontal, again at Amareleja and with $b=0.28$ (e.g.: files of $5 \times 1$ modules, each $1 \times \sqrt{ }\left(2 \mathrm{~m}^{2}\right), N_{\mathrm{TB}}=15 \times 1$. Practical reasons impose severe

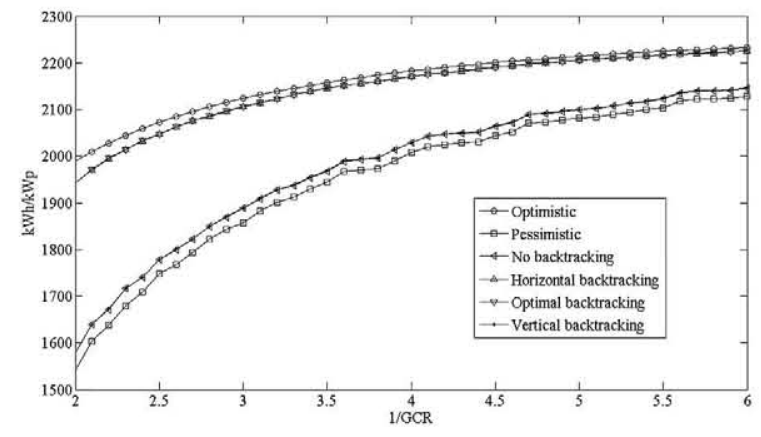

Figure 11. Energy yield versus the GCR for a two-axis tracking, primary horizontal at Amareleja with $b=0.28$ and NTB $=15 \times 1$. 
limits to $L_{\mathrm{NS}}$ values. Otherwise, the tracking structure occupation becomes very low and, due to the corresponding growth of required materials (steel and foundations), also very expensive. Here, we have set $L_{\mathrm{NS}}=0.58$. Again because of practical reasons, the rotation and tilt angles have also been limited to \pm 60 and $75^{\circ}$, respectively.

Table I gives numerical results for $1 / \mathrm{GCR}=5.5$. All these limits make this tracking type rather different than the primary vertical, and explain the lower energy collection for this 1/GCR value. A more detailed comparison is out of the scope of this work. However, concerning backtracking, a similar conclusion can be made: back-tracking is significantly more efficient on the primary axis than on the secondary one. Moreover, back-tracking on only the primary axis is likely to be a more optimal solution.

\section{CONCLUSIONS}

Back-tracking geometry for single-axis and two-axis tracking has been described and ideal tracking and back-tracking strategies have been compared in terms of energy yield for the representative cases. Numerical values have been presented for only a particular location and for grid connected PV plants. However, general considerations of the geometry governing Sun tracking lead us to propose the general validity of key results: that back-tracking is more attractive for the single horizontal axis than for the single vertical one and, concerning two axes, that backtracking is more efficient on the primary axis than on the secondary one.

Finally, because back-tracking avoids shade, its implementation in the current state-of-the-art of tracking technology is attractive not only for energy gain reasons but also for reducing the risk of hot-spots.

\section{ACKNOWLEDGEMENTS}

Reviewer 1 provided extremely lucid comments to the first version of this paper.

\section{REFERENCES}

1. Narvarte L, Lorenzo E. Tracking and Ground Cover Ratio. Progress in Photovoltaics: Research and Applications 2008; 16: 703-714.
2. Siemer J. An industry in transition. Global survey on tracking systems. Photon International 2009; 11: 138 189.

3. Kimber A, Mitchell L. Performance evaluation standards for large grid-connected PV systems in the United States and Germany. 20th European Photovoltaic Solar Energy Conference, 2005; 3229-3232.

4. Baumgartner FP, Büchel A, Bartholet R. Solar Wings: A new Lightweight PV Tracking System. 23rd European Photovoltaic Solar Energy Conference, 2008; $2790-2794$.

5. García M, Vera JA, Marroyo L, Lorenzo E, Pérez E. Solar-tracking PV plants in Navarra: A 10 MW assessment. Progress in Photovoltaics: Research and Applications 2009; 17: 337-346.

6. Lorenzo E, Macagnan MH. Considerations in the design of one axis tracking PV systems. Progress in Photovoltaics: Research and Applications 1994; 2: 45-55.

7. Lorenzo E, Pérez M, Ezpeleta A, Acedo J. Design of tracking photovoltaic systems with a single vertical axis. Progress in Photovoltaics: Research and Applications 2002; 10: 533-543.

8. Martínez-Moreno F, Muñoz J, Lorenzo E. Experimental model to estimate shading losses on PV arrays. Solar Energy Materials and Solar cells 2010; 94: 2298-2303.

9. Photovoltaic Geographical Information System (PVGIS): http://sunbird.jrc.it/pvgis/

10. Panico D, Garvison P, Wenger H, Shugar D. Backtracking: a novel strategy for tracking PV systems. 22nd IEEE Photovoltaic Specialist Conference, 1991; 668-673.

11. Lorenzo E, Maquedano C. Operational results of the $100 \mathrm{kWp}$ tracking PV plant at Toledo-PV project. $13 \mathrm{th}$ European Photovoltaic Solar Energy Conference, 1995; 2433-2436.

12. Muñoz J, Lorenzo E, Martinez-Moreno F, Marroyo L, Garcia M. An investigation into hot-spots in two large grid-connected PV plants. Progress in Photovoltaics: Research and Applications 2008; 16: 693-701.

13. SolarOptimus tracking system. (www.conergy. com). 